Revista de Matemática: Teoría y Aplicaciones 2(2): 1-7 (1995)

\title{
VALORES PROPIOS DE DIRICHLET ASOCIADOS A LA ECUACIÓN DE HILL CON POTENCIAL DE RUIDO BLANCO
}

\author{
Henry P. MCkean ${ }^{1}-$ SAntiago CAmbronero $^{2}$
}

\section{Resumen}

Mostramos que la ecuación de Hill con potencial de "ruido blanco" tiene una sucesión $\lambda_{n}$ de valores propios de Dirichlet que se comporta casi como en el caso clásico, en el sentido que $\lambda_{n}-n^{2} \pi^{2}$ posee un crecimiento logarítmico, proveniente de de una sucesión de variables gaussianas.

\begin{abstract}
We show that Hill's equation with white noise potential has a sequence of Dirichlet eigenvalues $\lambda_{n}$ that behaves almost like in the classical case, in the sense that $\lambda_{n}-n^{2} \pi^{2}$ has a logarithmic growth coming from a sequence of Gaussian random variables.
\end{abstract}

\section{Introducción}

La ecuación de Hill con potencial $Q$, y parámetro $\lambda$, está dada por

$$
-y^{\prime \prime}+Q y=\lambda y \text {. }
$$

Los valores propios de Dirichlet son aquellos valores de $\lambda$ para los cuales existe una solución no trivial de (1) satisfaciendo

$$
y(0)=y(1)=0 .
$$

En el caso clásico $Q$ es una función suave, y es sabido que (1) posee una sucesión de valores propios de Dirichlet $\lambda_{o}<\lambda_{1}<\ldots$, tal que $\lambda_{n}=n^{2} \pi^{2}+\int_{o}^{1} Q+O\left(\frac{1}{n^{2}}\right)$, para $n$ grande. De hecho, la cantidad de términos en esta expansión puede incrementarse indefinidamente, dependiendo del número de derivadas que $Q$ posea. Por ejemplo, si $Q$ es $\mathcal{C}^{2}$, entonces

$$
\lambda_{n}=n^{2} \pi^{2}+\int_{o}^{1} Q+\frac{c}{n^{2}}+O\left(\frac{1}{n^{3}}\right) .
$$

\footnotetext{
${ }^{1}$ Courant Institute of Mathematical Sciences, 251 Mercer Street, New York, NY 10012, Estados Unidos.

${ }^{2}$ Escuela de Matemática, Universidad de Costa Rica, 2060 San José, Costa Rica.
} 
Para revisar las demostraciones de dichos resultados, así como obtener más información al respecto, el lector puede consultar [2], [3], [7], y [8].

En el presente trabajo consideramos la ecuación (1), donde el potencial $Q$ es ruido blanco, esto es, la derivada formal de un movimiento Browniano, en algún espacio de probabilidad $(\Omega, \mathcal{B}, P)$. Una solución de esta ecuación es una función y de clase $\mathcal{C}^{1}$ que satisface la versión integral

$$
-y^{\prime}(x)+y^{\prime}(0)+\int_{0}^{x} y(t) d b(t)=\lambda \int_{0}^{x} y(t) d t
$$

donde $b$ es el correspondiente movimiento Browniano en $(\Omega, \mathcal{B}, P)$, y la integral de la izquierda se define como

$$
y(x) b(x)-\int_{0}^{x} b(t) y^{\prime}(t) d t .
$$

La solución $y$ con $y(0)=0, y^{\prime}(0)=1$ debe satisfacer entonces

$$
y(x)=\frac{\operatorname{sen} \sqrt{\lambda} x}{\sqrt{\lambda}}+\int_{o}^{x} \frac{\operatorname{sen} \sqrt{\lambda}(x-t)}{\sqrt{\lambda}} y(t) d b,
$$

o equivalentemente

$$
y(x)=\frac{\operatorname{sen} \sqrt{\lambda} x}{\sqrt{\lambda}}-\int_{o}^{x} b(t)\left[\frac{\operatorname{sen} \sqrt{\lambda}(x-t)}{\sqrt{\lambda}} y^{\prime}(t)-\cos \sqrt{\lambda}(x-t) y(t)\right] d t .
$$

Si iteramos esta ecuación obtenemos

$$
y=y_{o}+y_{1}+\ldots+y_{n}+R_{n}(x),
$$

donde $y_{o}=\frac{\operatorname{sen} \sqrt{\lambda} x}{\sqrt{\lambda}}, y_{n+1}=\int_{o}^{x} \frac{\operatorname{sen} \sqrt{\lambda}(x-t)}{\sqrt{\lambda}} y_{n}(t) d b(t)$.

Esto sugiere que definamos $y=\sum_{n=0}^{\infty} y_{n}(x)$, para lo cual trataremos de estimar $\left|y_{n}\right| \mathrm{y}$ mostrar convergencia. $|y|$ denotará la norma sup de $y$ en $[0,1]$. Los siguientes resultados serán de gran utilidad.

Lema 1. Si $G_{o} \equiv a \geq 0$, y para todo $n=0,1, \ldots$

$$
G_{n+1}(x)=2 c \int_{0}^{x} G_{n}(t) d t+\frac{c}{\mu} G_{n}(x),
$$

donde $c, \mu>0$, entonces $G_{n}$ está dado por

$$
G_{n}(x)=a(2 c)^{n} \sum_{k=0}^{n} a(n, k) x^{k} \mu^{-(n-k)},
$$


donde $a(n, 0)=2^{-n}, \mathrm{y} \quad a(n, k)=\frac{n(n-1) \cdots(n-k+1)}{(k !)^{2} 2^{n-k}}$, para $k=1, \ldots, n$.

PRUEBA

Basta observar que $a(n+1, k)=\frac{1}{k} a(n, k-1)+\frac{1}{2} a(n, k)$, y usar inducción en $n$.

Lema 2. Supongamos que $\left(f_{n}\right)$ y $\left(g_{n}\right)$ satisfacen

$$
\begin{gathered}
0 \leq f_{n+1}(x) \leq c \int_{0}^{x}\left(f_{n}+g_{n}\right) \\
0 \leq g_{n+1}(x) \leq c \int_{0}^{x}\left(f_{n}+g_{n}\right)+\frac{c}{\mu} f_{n}(x),
\end{gathered}
$$

para $n=0,1, \ldots, x \in[0,1], \mathrm{y}$ definamos $h_{n}=\max \left(f_{n}, g_{n}\right)$. Entonces, para $x \in[0,1], \mathrm{y}$ $\mu>2 c$ tenemos

$$
\sum_{n=0}^{\infty} h_{n}(x) \leq 2 e^{4 c}\left|h_{o}\right|
$$

Prueba

Nótese que $h_{n+1}(x) \leq 2 c \int_{o}^{x} h_{n}+\frac{c}{\mu} h_{n}(x)$. Tomando $a=\left|h_{o}\right|$, el lema anterior permite concluir que

$$
\begin{aligned}
\sum_{n=0}^{\infty} h_{n}(x) & \leq \sum_{n=0}^{\infty} G_{n}(x) \\
& \leq \sum_{n=0}^{\infty} a(2 c)^{n} \sum_{k=0}^{n} \frac{n(n-1) \cdots(n-k+1)}{(k !)^{2} 2^{n-k} \mu^{n-k}} \\
& =a \sum_{k=0}^{\infty} \frac{(2 c)^{k}}{(k !)^{2}} \sum_{n=k}^{\infty} n(n-1) \cdots(n-k+1)\left(\frac{c}{\mu}\right)^{n-k} \\
& =a \sum_{k=0}^{\infty} \frac{(2 c)^{k}}{k !}\left(1-\frac{c}{\mu}\right)^{-(k+1)} \\
& \leq 2 a \sum_{k=0}^{\infty} \frac{(4 c)^{k}}{k !}=2 a e^{4 c} .
\end{aligned}
$$

Es fácil mostrar que $f_{n}=\left|y_{n_{o}+n}\right|$, y $g_{n}=\frac{1}{\mu}\left|y_{n_{o}+n}^{\prime}\right|$ satisfacen las hipótesis del lema 2, con $\mu=\sqrt{\lambda}$, y $c=|b|$. Como consecuencia obtenemos que

$$
\sum_{n=n_{o}}^{\infty}\left|y_{n}\right| \leq 2 a e^{4|b|}, \quad \sum_{n=n_{o}}^{\infty}\left|y_{n}^{\prime}\right| \leq 2 a \mu e^{4|b|},
$$

para $\mu>2|b|$, donde $a=\operatorname{máx}\left\{\left|y_{n_{o}}\right|, \frac{1}{\mu}\left|y_{n_{o}}^{\prime}\right|\right\}$. De la definición de $y_{n}$ se sigue inmediatamente el siguiente teorema. 
Teorema 1 Para $\lambda>4|b|^{2}$, la solución de (1), con condiciones $y(0)=0, y^{\prime}(0)=1$, está dada por $y=\sum y_{n}$, donde la convergencia es uniforme en $[0,1]$. También tenemos que $y^{\prime}=\sum y_{n}^{\prime}$, uniformemente en $[0,1]$.

Observación. El lema 2 muestra también que el orden de $\left|R_{n}\right|$ está dominado por el orden de máx $\left\{\left|y_{n}\right|, \frac{1}{\mu}\left|y_{n}^{\prime}\right|\right\}$. O sea que si mostramos que máx $\left\{\left|y_{n}\right|, \frac{1}{\mu}\left|y_{n}^{\prime}\right|\right\}=O\left(\mu^{\alpha}\right)$, entonces $\left|R_{n}\right|=O\left(\mu^{\alpha}\right)$. En lo que sigue vamos a usar este hecho para estimar los valores propios $\lambda_{n}$.

Observe que $\left|y_{o}\right| \leq \mu^{-1}$, y $\left|y_{o}^{\prime}\right| \leq 1$. Por otro lado, de la definición tenemos $y_{1}=$ $\int_{o}^{x} b(t) \frac{\operatorname{sen} \mu(2 t-x)}{\mu} d t=\frac{1}{\mu} \operatorname{Im}\left(e^{-i \mu x} I_{1}(x)\right)$, donde

$$
I_{1}=\int_{0}^{x} b(t) e^{2 i \mu t} d t=-\int_{\frac{\pi}{2 \mu}}^{x+\frac{\pi}{2 \mu}} b\left(t-\frac{\pi}{2 \mu}\right) e^{2 i \mu t} d t .
$$

Si sumamos estas dos integrales, vemos que $2 I_{1}$ es igual a

$$
\int_{x}^{x+\frac{\pi}{2 \mu}} b\left(t-\frac{\pi}{2 \mu}\right) e^{2 i \mu t}-\int_{0}^{\frac{\pi}{2 \mu}} b(t) e^{2 i \mu t}+\int_{\frac{\pi}{2 \mu}}^{x}\left[b\left(t-\frac{\pi}{2 \mu}\right)-b(t)\right] e^{2 i \mu t} .
$$

Usando esta expresión, y el módulo de continuidad de $b(t)$, obtenemos

$$
\begin{aligned}
\left|I_{1}\right| & \leq \frac{\pi|b|}{2 \mu}+\frac{1}{2} \max \left\{\left|b\left(t-\frac{\pi}{2 \mu}\right)-b(t)\right|: \frac{\pi}{2 \mu} \leq t \leq x\right\} \\
& \leq \frac{\pi|b|}{2} \mu^{-1}+\mu^{-1 / 2}(\log \mu)^{1 / 2} \leq 2 \mu^{-1 / 2}(\log \mu)^{1 / 2},
\end{aligned}
$$

para $\mu \geq \mu_{o}(w)$, y casi todo $w$.

Esto implica que $\left|y_{1}\right| \leq 2 \mu^{-3 / 2}(\ln \mu)^{1 / 2}$, para casi todo $w$, donde $\mu \geq \mu_{o}(w)$. Similarmente tenemos que $\frac{1}{\mu}\left|y_{1}^{\prime}\right| \leq 3 \mu^{-3 / 2}(\ln \mu)^{1 / 2}$, para $\mu \geq \mu_{o}$.

De la observación hecha arriba se sigue que, para $\lambda$ grande,

$$
y(x, \lambda)=\frac{\operatorname{sen} \sqrt{\lambda} x}{\sqrt{\lambda}}+O\left(\lambda^{-3 / 4+}\right)
$$

y entonces $y(1, \lambda)=0$ implica $\sqrt{\lambda}=n \pi+\varepsilon_{n}$, donde $\varepsilon_{n}=O\left(n^{-1 / 2+}\right)$. Recíprocamente, para $n$ grande, existe $\varepsilon_{n}$ tal que $y\left(1,\left(n \pi+\varepsilon_{n}\right)^{2}\right)=0$, y $\varepsilon_{n}=O\left(n^{-1 / 2+}\right)$.

Los siguientes resultados nos permitirán obtener una expresión más exacta para $\varepsilon_{n}$.

Lema 3. Sea $X_{n}(t)=\int_{o}^{t} \cos (2 n \pi u) d b(u)$. Cuando $n \rightarrow \infty$ tenemos $\left|X_{n}\right|=O\left(n^{0+}\right)$, con probabilidad 1.

Prueba: 
Cada $X_{n}(t)$ es una martingala, con $E X_{n}^{2 p}(1) \leq C_{p}$, para $p \geq 1$, y entonces $P\left(\operatorname{máx}_{0 \leq t \leq 1}\left|X_{n}(t)\right| \geq n^{\alpha}\right) \leq \frac{C_{p}}{n^{2 p \alpha}}$ (ver [5], páginas 13 y 163$)$.

Dado $\alpha>0$, podemos escoger $p \geq 1$ tal que $2 p \alpha>1$, y entonces, por Borel-Cantelli,

$$
P\left(\operatorname{máx}\left|X_{n}(t)\right| \leq n^{\alpha} \text {, cuando } n \uparrow \infty\right)=1 \text {, }
$$

para todo $\alpha>0$. Como podemos restringirnos a valores racionales de $\alpha$, concluimos que

$$
P\left(\operatorname{máx}\left|X_{n}(t)\right|=O\left(n^{\alpha}\right), n \uparrow \infty, \forall \alpha>0\right)=1 .
$$

Corolario 1. $\operatorname{máx}_{0 \leq t \leq 1}\left|\int_{o}^{t} b(u) \operatorname{sen}(2 n \pi u) d u\right|=O\left(n^{-1+}\right)$, cuando $n \rightarrow \infty$, con probabildad 1.

Prueba:

$\int_{o}^{t} b(u) \operatorname{sen}(2 n \pi u) d u=\frac{1}{2 n \pi} \int_{o}^{t} \cos (2 n \pi u) d b-\frac{b(t) \cos (2 n \pi t)}{2 n \pi}$.

El mismo argumento muestra el siguiente lema.

Lema 4. Sea $Z_{n}(t)=\int_{o}^{t} u b(u) \cos (2 n \pi u) d u$. Cuando $n \rightarrow \infty$ tenemos que $\left|Z_{n}\right|=$ $O\left(n^{-1+}\right)$, con probabilidad 1 .

Observación. Resultados similares son válidos si intercambiamos los roles de seno y coseno, con obvias modificaciones.

Lema 5. Sea $X_{n}(t)=\int_{o}^{t} b(u) \cos (2 n \pi u) c(u) d u$, donde $c(\cdot)$ está dado por

$c(t)=\int_{o}^{t} b(u) \operatorname{sen}(2 n \pi u) d u$. Cuando $n \rightarrow \infty$ tenemos que $X_{n}(t)=-\frac{1}{4 n \pi} \int_{o}^{t} b^{2}(u) d u+$ $O\left(n^{-2+}\right)$. Más precisamente

$$
\operatorname{máx}_{0 \leq t \leq 1}\left|X_{n}(t)+\frac{1}{4 n \pi} \int_{o}^{t} b^{2}(u) d u\right|=O\left(n^{-2+}\right),
$$

con probabilidad 1 .

Prueba:

Observemos primero que $c(t)=\frac{1}{2 n \pi}[e(t)-b(t) \cos 2 n \pi t]$, con $e(t)=\int_{o}^{t} \cos (2 n \pi u) d b, \mathrm{y}$ entonces

$$
E\left[c^{2 p}(t)\right] \leq \frac{2^{2 p}}{(2 n \pi)^{2 p}}\left[E e^{2 p}(t)+E b^{2 p}(t)\right] \leq \frac{C_{p}}{n^{2 p}},
$$

donde $C_{p}$ es una constante que depende sólo de $p$. Por otro lado, como $|c|=O\left(n^{-1+}\right)$, tenemos que

$$
X_{n}(t)=\int_{o}^{t} \frac{(\cos (4 n \pi u)-1)}{4 n \pi} b^{2}(u) d u-\frac{1}{2 n \pi} Y_{n}(t)+O\left(n^{-2+}\right)
$$

donde $Y_{n}(t)=\int_{o}^{t} c(u) \operatorname{sen}(2 n \pi u) d b$ satisface $E Y_{n}^{2 p}(t) \leq \frac{\hat{C}_{p}}{n^{2 p}}$, y por lo tanto $P\left[\operatorname{máx}_{0 \leq t \leq 1}\left|Y_{n}(t)\right|>n^{\alpha}\right] \leq \frac{1}{n^{2 p \alpha}} E Y_{n}^{2 p}(1) \leq \frac{C_{p}}{n^{2 p \alpha+2 p}}$. 
Por Borel-Cantelli se sigue que $\left|Y_{n}\right|=O\left(n^{-1+}\right)$.

Similarmente tenemos que $\int_{o}^{t} \cos (4 n \pi u) b^{2}(u) d u=O\left(n^{-1+}\right)$.

Volvamos ahora al problema de aproximar $\lambda_{n}$. Como observamos anteriormente, $\sqrt{\lambda_{n}}=$ $n \pi+\varepsilon_{n}$, donde $\varepsilon_{n}=O\left(n^{-1 / 2+}\right)$, y entonces $(-1)^{n} \sqrt{\lambda_{n}} y_{1}\left(1, \lambda_{n}\right)$ se puede expresar como

$$
\operatorname{sen} \varepsilon_{n} \int_{0}^{1} b(t) \cos \left(2 \sqrt{\lambda_{n}} t\right) d t+\cos \varepsilon_{n} \int_{0}^{1} b(t) \operatorname{sen}\left(2 \sqrt{\lambda_{n}} t\right) d t .
$$

Por el corolario 1 es fácil concluir que $\sqrt{\lambda_{n}} y_{1}\left(1, \lambda_{n}\right)=O\left(n^{-1+}\right)$, y lo mismo es válido para $y_{1}^{\prime}\left(1, \lambda_{n}\right)$. Como consecuencia $\varepsilon_{n}=O\left(n^{-1+}\right)$.

Para obtener un mejor estimado de $\varepsilon_{n}$ necesitamos acotar $y_{2}\left(1, \lambda_{n}\right)$. Usando el hecho que

$$
y_{2}=-\int_{o}^{x} b(t)\left[\frac{\operatorname{sen} \sqrt{\lambda}(x-t)}{\sqrt{\lambda}} y_{1}^{\prime}(t)-\cos \sqrt{\lambda}(x-t) y_{1}(t)\right] d t,
$$

se obtiene que $y_{2}=A+B+C+D$, con

$$
\begin{aligned}
& A=\frac{\operatorname{sen} \sqrt{\lambda} x}{2 \sqrt{\lambda}}\left[\left(\int_{o}^{x} b(t) \cos 2 \sqrt{\lambda} t d t\right)^{2}+\left(\int_{o}^{x} b(t) \operatorname{sen} 2 \sqrt{\lambda} t\right)^{2}\right], \\
& B=-\frac{\cos \sqrt{\lambda} x}{\sqrt{\lambda}}\left(\int_{o}^{x} b(t) \cos 2 \sqrt{\lambda} t\right)\left(\int_{o}^{x} b(t) \operatorname{sen} 2 \sqrt{\lambda} t\right) \\
& C=2 \frac{\cos \sqrt{\lambda} x}{\sqrt{\lambda}}\left[\int_{o}^{x} b(t) \cos 2 \sqrt{\lambda} t d t \int_{o}^{t} b(u) \operatorname{sen} 2 \sqrt{\lambda} u d u\right] \\
& D=\frac{\cos \sqrt{\lambda} x}{2 \lambda} \int_{o}^{x} b^{2}(t) d t-\frac{1}{2 \lambda} \int_{o}^{x} b^{2}(t) \cos \sqrt{\lambda}(x-2 t) d t
\end{aligned}
$$

De lo anterior, y los lemas 3,4 y 5 , concluimos que $y_{2}\left(1, \lambda_{n}\right)=O\left(n^{-3+}\right)$, y entonces

$$
0=(-1)^{n} \sqrt{\lambda_{n}} y\left(1, \lambda_{n}\right)=\varepsilon_{n}+\int_{o}^{1} b(t) \operatorname{sen}(2 n \pi t) d t+O\left(n^{-2+}\right)
$$

esto es

$$
\varepsilon_{n}=-\int_{o}^{1} b(t) \operatorname{sen}(2 n \pi t) d t+O\left(n^{-2+}\right)=\frac{1}{2 n \pi}\left(b(1)-X_{n}\right)+O\left(n^{-2+}\right),
$$

donde

$$
X_{n}=\int_{o}^{1} \cos (2 n \pi t) d b(t) .
$$

Hemos mostrado el siguiente teorema.

Teorema 2 Los valores propios de Dirichlet asociados al operador $-\frac{d^{2}}{d x^{2}}+Q$, donde $Q$ es ruido blanco, están dados por

$$
\lambda_{n}=n^{2} \pi^{2}+b(1)-\int_{o}^{1} \cos (2 n \pi t) d b(t)+O\left(n^{-1+}\right),
$$

para valores grandes de $n$, donde b es el movimiento Browniano correspondiente. 
Nótese la diferencia con respecto al caso clásico (cuando $Q$ es una función suave). Aquí obtenemos la sucesión de variables gausianas $X_{n}$, que satisface $X_{n}=O\left(n^{0+}\right)$. De hecho, no es difícil mostrar que máx $\left\{\left|X_{k}\right|: k=0, \ldots, n\right\}$ está dominado por $\sqrt{3 \log n}$, para valores grandes de $n$. Para esto basta observar que $E X_{n}=0, E X_{n}^{2}=\frac{1}{2}$, y $E X_{n} X_{m}=0$, para $n \neq m$, y por lo tanto las variables Gausianas $X_{n}$ son independientes e idénticamente distribuidas. Esto implica que

$$
\begin{aligned}
P\left[\operatorname{máx}_{k=1, \ldots, n}\left|X_{k}\right| \leq \sqrt{\alpha \ln n}\right] & =\left\{P\left[\left|X_{1}\right| \leq \sqrt{\alpha \ln n}\right]\right\}^{n} \\
& =\left\{1-\frac{e^{-\alpha \ln n}}{\sqrt{\pi \alpha \ln n}}(1+o(1))\right\}^{n} \\
& =\exp \left[-n^{1-\alpha}(\pi \alpha \ln n)^{-1 / 2}(1+o(1))\right],
\end{aligned}
$$

y por lo tanto

$$
\begin{aligned}
P\left[\operatorname{máx}_{k=1, \ldots, n}\left|X_{k}\right|>\sqrt{\alpha \ln n}\right] & =1-\exp \left[-n^{1-\alpha}(\pi \alpha \ln n)^{-1 / 2}(1+o(1))\right] \\
& =\frac{1}{n^{\alpha-1} \sqrt{\pi \alpha \ln n}}(1+o(1)) \\
& \leq \frac{2}{n^{\alpha-1}}
\end{aligned}
$$

para $n$ grande. Por Borel Cantelli se sigue que

$$
P\left[\operatorname{máx}_{k=1, \ldots, n}\left|X_{k}\right| \leq \sqrt{\alpha \ln n}, n \uparrow \infty\right]=1,
$$

para todo $\alpha>2$.

\section{Referencias}

[1] Breiman, L. (1992) Probability. Classics in Appl. Math. SIAM.

[2] Eastham, M.S.P. (1971) The Spectral Theory of Periodic Differential Equations. Scottish Academic Press.

[3] Hochstadt, H. (1961) "Asymptotic Estimates for the Sturm - Liouville Spectrum", Com. Pure Appl., vol. XIV, 749-764.

[4] Itô, K. \& Mckean, H. (1974) Diffusion Processes and their Sample Paths. Segunda edición, Springer-Verlag.

[5] Karatzas, I. \& Shreve, S.E. (1991) Brownian Motion and Stochastic Calculus. SpringerVerlag. Segunda edición.

[6] Mckean, H. (1969) Stochastic Integrals. Academic Press, New York. 
[7] Mckean, H. \& Moerbeke, P. (1975) "The Spectrum of Hill's Equation", Inv. Math. 30, 217-274.

[8] Levitan, B.M. \& Sargsjar, I.S. (1991) Sturm Liouville and Dirac Operators. Kluwer Academic Publishers. 